\title{
VASECTOMÍA SIN BISTURÍ: RESULTADOS DE UNA COHORTE DE 309 PACIENTES*
}

\author{
Drs. Francisco Sepúlveda T. ${ }^{1}$, Daniela Fleck L. ${ }^{2}$, Josvany Sánchez. ${ }^{3}$, \\ Alejandro Mercado C. ${ }^{1}$, Diego Reyes O. ${ }^{1}$, Juan P. Hidalgo V. ${ }^{1}$, Cristián Palma C. ${ }^{1,2,3}$
}

\author{
Servicio de Urología. Hospital Clínico Universidad de Chile, Santiago, Chile. \\ Servicio de Urología, Clínica Las Condes, Santiago, Chile. \\ ${ }^{3}$ Departamento de Andrología. Fundació Puigvert, Barcelona, España.
}

\begin{abstract}
No-scalpel vasectomy

Introduction: Vasectomy is a safe and effective technique of male fertility control. Despite this, in the world are carried out more than double female sterilization in comparison with vasectomies, that is more pronounced in less developed countries. Aims: To present our experience and results in patients undergoing a no-scalpel vasectomy. Methods and Material: A total of 309 patients undergoing a no-scalpel vasectomy between June 2009 and May 2010 were included. For each case was record age, operative time, sperm count at 3 months post vasectomy and peri-operative morbidity. Results: 309 vasectomies were performed, 281 patients $(91 \%)$ were controlled with at least one sperm count. Azoospermia was obtained in the first sperm count at 3 months in 189 patients (67\%). In 81 patients (29\%) were observed $\leq 100,000$ sperm $100 \%$ immobile. 9 patients $(3.2 \%)$ needed a second semen analysis and 2 patients a third one for less than 100,000 sperm that were $100 \%$ immobile. 6 patients $(2 \%)$ consulted by minor complications such as postoperative pain, epididymitis or hematoma with spontaneous resolution. Conclusions: Vasectomy is a safe and reproducible method of male contraception, presenting an effectiveness rates higher than others contraceptive methods. There are no absolute contraindications for performing the procedure. A sperm count should be done at 3 months of the procedure. The early failure rate in our study is $0.3 \%$.
\end{abstract}

Key words: Contraceptive Agents, vasectomy, azoospermia, sperm count.

\section{Resumen}

Introducción: La vasectomía es una técnica segura y efectiva para el control de la fertilidad masculina. A pesar de esto, en el mundo se realizan el doble de esterilizaciones femeninas respecto a vasectomías, lo cual es más acentuado en países en vías de desarrollo. Objetivos: El objetivo de este trabajo es presentar nuestra experiencia y resultados en pacientes sometidos a una vasectomía sin bisturí. Materiales y Métodos: Se incluyó un total de 309 pacientes sometidos a una vasectomía sin bisturí entre junio de 2009 y mayo de

* Recibido el 2 de agosto de 2013 y aceptado para publicación el 21 de octubre de 2013.

Los autores no refieren conflictos de interés.

Correspondencia: Dr. Cristian Palma C.

Av. Santos Dumont 999, Codigo Postal 8380456, Santiago, Chile. cpalma@clc.cl 
2010. En cada caso se evaluó la edad, tiempo operatorio, espermiograma a los 3 y 6 meses (en caso de ser necesario) y la morbilidad perioperatoria. Resultados: 281 pacientes (91\%) se controlaron con al menos un espermiograma. En 189 pacientes (67\%) se evidenció azoospermia en el espermiograma a los 3 meses. En 81 pacientes $(29 \%)$ se evidenció un recuento con $\leq 100.000$ espermatozoides $100 \%$ inmóviles. En 9 pacientes $(3,2 \%)$ se necesitó un segundo espermiograma para obtener menos de 100.000 espermatozoides $100 \%$ inmóviles. Seis pacientes $(2 \%)$ consultaron por complicaciones menores: orquialgia, epididimitos o hematoma del sitio operatorio. A 2 años de seguimiento, no se registraron embarazos. Conclusiones: La vasectomía sin bisturí es un método seguro y reproducible de anticoncepción masculina, presentando tasas de efectividad superiores al resto de los métodos anticonceptivos. Un espermiograma de control debe ser realizado a los 3 meses de realizado el procedimiento. La tasa de falla temprana se estima en $0,3 \%$ de los pacientes.

Palabras clave: Métodos anticonceptivos, vasectomía, azoospermia, espermiograma.

\section{Introducción}

La vasectomía es un método seguro y efectivo de anticoncepción masculina. Su uso actual se estima en más de 30 millones de hombres en el mundo ${ }^{1-4}$. Sin embargo, en el mundo se realizan más del doble de esterilizaciones femeninas respecto a la vasectomía ${ }^{1,2}$.

A pesar del bajo riesgo de embarazo posterior al procedimiento $^{5-7}$, se recomienda la realización de un espermiograma a los 3 meses de operado el paciente. Hasta entonces se deben utilizar otros métodos anticonceptivos. En caso de evidenciarse azoospermia o un recuento $\leq 100.000$ espermatozoides $100 \%$ inmóviles el paciente puede ser dado de alta ${ }^{8-12}$.

El siguiente trabajo tiene por objetivo mostrar nuestros resultados en pacientes sometidos a una vasectomía sin bisturí además de una revisión de la literatura.

\section{Materiales y Métodos}

Se realizó un estudio retrospectivo de 309 pacientes sometidos a una Vasectomía sin bisturí entre junio del año 2009 a mayo del año 2010 en la Fundació Puigvert (Barcelona, España) y el Hospital Clínico de la Universidad de Chile. Previo a la cirugía se obtuvo un consentimiento informado en cada paciente en el cual se explicaron los resultados de otros métodos anticonceptivos y sus tasas de éxito, además de las complicaciones tempranas y tardías de la cirugía. Si bien la vasectomía se planteó como un método irreversible, se explicó la posibilidad de una vaso-vasostomía y sus tasas de éxito.

Todos los procedimientos fueron realizados por dos cirujanos (CP, JS) utilizando la técnica de vasectomía sin bisturí descrita por Li et al en $1974^{13}$. Se inyectan $5 \mathrm{cc}$ de lidocaína al $2 \%$ en rafe medio escrotal para la anestesia de ambos cordones espermáticos. Posteriormente, con pinza de disección se realiza pequeña divulsión de piel en sitio punción anestésica y se exterioriza conducto deferente dere- cho previamente identificado el que se toma con pinza de anillo. Se procede a sección, ligadura (vicryl 2-0) y electrocoagulación de extremos de conducto deferente, además de la interposición de fascias. Se regresa conducto deferente al lecho escrotal y se replica procedimiento a izquierda.

En todos los casos se indicó reposo el día de la cirugía, lo que se prolongó por 5 días en aquellos pacientes con un trabajo que involucrase esfuerzo físico. En todos los pacientes se registró el tiempo operatorio y las complicaciones tempranas y tardías.

Se realizó un control médico a los 10 días de realizado el procedimiento en todos los pacientes. El primer espermiograma fue realizado a los 3 meses posteriores a la vasectomía y luego, con un intervalo de 6 semanas hasta obtener un recuento $\leq 100.000$ espermatozoides $100 \%$ inmóviles. La concentración de espermatozoides, el volumen del eyaculado y el número de espermatozoides inmóviles se incluyó en cada reporte. Todos los espermiogramas fueron evaluados en un laboratorio de infertilidad. En caso de evidenciarse azoospermia, se procedió a centrifugar las muestras para confirmar esta condición.

A dos años de realizado el procedimiento, todos los pacientes fueron contactados vía telefónica para evaluar la presencia de embarazo posterior a la vasectomía.

\section{Resultados}

Durante el período de estudio se realizó un total de 309 vasectomías sin bisturí. El promedio de edad fue de 39,5 (25-57) años. El tiempo promedio operatorio fue de 15,4 (8-35) minutos.

En 281 pacientes $(91 \%)$ se realizó un control con al menos un espermiograma. Un total de 28 pacientes faltaron al primer control, excluyéndose de los resultados del estudio. En 189 pacientes (67\%) se obtuvo azoospermia en el primer espermiograma a los 3 meses, mientras que en 81 pacientes (29\%) se obtuvo un recuento $\leq 100.000$ espermatozoides $100 \%$ inmóviles en el mismo control. Nueve pacien- 
Tabla 1. Resultados de espermiogramas

\begin{tabular}{|lccc|}
\hline & n pacientes $(\%)$ & n pacientes $(\%)$ & n pacientes $(\%)$ \\
4 meses & 3 meses & $193(69)$ & $193(69)$ \\
\hline Azoospermia & $189(67)$ & $85(30)$ & $87(31)$ \\
$<100.000$ espermatozoides $100 \%$ inmóviles & $81(29)$ & $2(1)$ & 0 \\
$>100.000$ espermatozoides $100 \%$ inmóviles & $9(3)$ & 0 & 0 \\
Espermatozoides móviles & $2(1)^{*}$ & $280^{\dagger}$ & $280 \dagger$ \\
Total & 281 & 19 & \\
\hline
\end{tabular}

*1 paciente con $80 \%$ de espermatozoides móviles, se indica nueva vasectomía. “excluyendo al paciente con una nueva vasectomía.

tes $(3,2 \%)$ requirieron un segundo espermiograma 4 meses posterior a la cirugía y dos pacientes un tercer espermiograma a los 6 meses de realizada la cirugía para obtener un recuento $\leq 100.000$ espermatozoides $100 \%$ inmóviles. En dos pacientes se detectó la presencia de espermatozoides móviles en el primer espermiograma de control, evidenciándose en uno de estos pacientes $(0,3 \%)$ una doble ligadura del mismo conducto deferente (falla temprana) motivo por el cual se decidió realizar una nueva vasectomía. El otro paciente cumplió criterios de alta en el segundo espermiograma. Las características de los espermiogramas se muestran en la Tabla 1.

Seis pacientes $(2 \%)$ consultaron por complicaciones menores, las que se detallan en la Tabla 2. De estos, un paciente con epididimitis fue tratado con antibióticos por vía oral hasta completar 14 días de tratamiento y el resto de los pacientes con antiinflamtorios por 5 días además de los indicados inicialmente. En todos los casos se indicó suspensión escrotal. Para el control de los 3 meses se evidenció una remisión completa de los síntomas en todos los pacientes. Después de 2 años de realizado el procedimiento todos los pacientes con al menos un control postoperatorio fueron controlados vía telefónica. Ningún paciente refirió embarazo en alguna de sus parejas.

\section{Discusión}

La vasectomía es un método anticonceptivo permanente, reproducible y efectivo. Actualmente, es el procedimiento urológico más frecuentemente realizado en Estados Unidos, con más de 600.000 casos al año ${ }^{1-4}$.

Existen múltiples formas de acceder al conducto deferente, sin embargo, la técnica más utilizada es la de la Vasectomía sin bisturí descrita por $\mathrm{Li}^{13}$. A pesar de que la técnica de acceso no afecta el éxito o
Tabla 2. Complicaciones

\begin{tabular}{|lcc|}
\hline Complicación & n & $(\mathbf{\%})$ \\
\hline Dolor postoperatorio escrotal & 4 & $(1,2)$ \\
\hline Epididimitis & 1 & $(0,3)$ \\
Hematoma & 1 & $(0,3)$ \\
\hline
\end{tabular}

falla de este procedimiento, la técnica sin bisturí está asociada a una menor incidencia de complicaciones como hematoma escrotal e infecciones de la herida operatoria además de asociarse a un menor dolor postoperatorio ${ }^{4,7,12-14}$. En nuestra serie no se observaron infecciones de herida operatoria.

El espermiograma es fundamental en el seguimiento de estos pacientes y debe ser realizado idealmente a los 3 meses de realizado el procedimiento, período en el cual debiesen ocurrir al menos 20 eyaculaciones ${ }^{7,12}$. Se describe que hasta un $30 \%$ de los pacientes no asistirán a este primer control postoperatorio con un espermiograma, lo cual no se evidenció en nuestra serie ya que un $90 \%$ de los pacientes asistieron con un espermiograma a los 3 meses. Esto podría explicarse debido a la aplicación rigurosa de un consentimiento informado preoperatorio donde se refuerza la importancia del control con espermiograma para declarar infértil al paciente.

La depuración de los espermatozoides residuales en la Ampulla del conducto deferente y las vesículas seminales es variable, por lo que al momento no existe un claro consenso sobre cuando debe obtenerse el primer espermiograma y cuantas muestras debiesen solicitarse; sin embargo, la mayoría de los protocolos utilizados en diferentes instituciones solicitan una muestra a los 3 meses de realizado el procedimiento ya que esto reduciría la tasa de falsos positivos, disminuyendo así el número de esper- 
miogramas innecesarios ${ }^{8,9}$. La utilización de otros métodos anticonceptivos puede abandonarse si el espermiograma muestra azoospermia o un recuento $\leq 100.000$ espermatozoides $100 \%$ inmóviles ${ }^{7,12}$. Es importante recalcar que entre un 10 y un $40 \%$ de los pacientes presentará espermatozoides inmóviles en el primer espermiograma a los 3 meses $^{7,15,16}$. En nuestra serie el $29 \%$ de los pacientes presentó un espermiograma con un recuento $\leq 100.000$ espermatozoides $100 \%$ inmóviles. Múltiples estudios han demostrado que el riesgo de embarazo no es superior respecto a aquellos pacientes en los que se evidencia azoospermia ${ }^{7,9,12}$. Estos espermatozoides pueden aparecer incluso hasta un año posterior a la realización de la vasectomía, por lo que no sería necesario repetir el estudio en estos casos. En nuestra serie el 31\% de los pacientes fue dado de alta con este criterio.

La falla temprana del procedimiento, ya sea por recanalización temprana o debido a una doble ligadura del mismo conducto deferente, se describe en un $0,2-5,3 \%$ de los casos y está relacionada con la experiencia del cirujano y la técnica quirúrgica utilizada $^{12,13}$. La electrocoagulación de ambos extremos del conducto deferente con la interposición de fascia son las únicas medidas que han mostrado una disminución del riesgo de falla de la vasectomía (presencia de espermatozoides móviles o un recuento mayor a 100.000 espermatozoides en el eyaculado) después de 3-6 meses $^{12-15}$. En nuestra serie, un paciente $(0,3 \%)$ presentó un $80 \%$ de espermatozoides móviles en el espermiograma de control a los 3 meses. El examen físico reveló una doble ligadura del conducto deferente en un lado y la indemnidad del conducto deferente contralateral.

La falla tardía, definida como la aparición de espermatozoides móviles en un paciente que cumplió con criterios de alta, ocurre en 1 de cada 2.000 pacientes ${ }^{7,11,12,17}$. Este tipo de falla es debido a una recanalización del conducto deferente y tiende a identificarse debido a un embarazo. El espermiograma conforma la reaparición de espermatozoides móviles. Al comparar la tasa de falla de la vasectomía con la de los otros métodos anticonceptivos se puede evidenciar la superioridad de esta técnica respecto a la esterilización femenina y a los anticonceptivos orales, siendo solo superada en efectividad por los implantes de Levonorgestrel ${ }^{18}$.

En el caso de un espermiograma a los 3 meses con espermatozoides móviles o una concentración mayor a 100.000 espermatozoides, el espermiograma debe repetirse con un intervalo de 4 a 6 semanas hasta lograr una concentración $\leq 100.000$ espermatozoides 100\% inmóviles. En caso de persistir con una alta concentración a los 6 meses de realizado el procedimiento, se recomienda repetir la vasectomía.

\section{Conclusiones}

La vasectomía sin bisturí es un método anticonceptivo seguro y reproducible, presentando una efectividad superior al resto de los métodos anticonceptivos y con una baja tasa de complicaciones. Al ser comparada con su contraparte femenina, tiene la ventaja de ser un procedimiento ambulatorio con una morbilidad perioperatoria baja, especialmente si se utiliza la técnica sin bisturí. La interposición de fascia espermática y electrocoagulación de los extremos del conducto deferente se asocian a un menor riesgo de falla del procedimiento.

Un espermiograma debe ser realizado a los 3 meses post procedimiento. Aquellos pacientes con azoospermia o con un recuento menor o igual a 100.000 espermatozoides $100 \%$ inmóviles pueden abandonar otros métodos anticonceptivos y ser dados de alta. En nuestra experiencia la falla temprana del procedimiento ocurre en un $0,3 \%$ de los pacientes. La falla tardía se estima en 1 de cada 2.000 pacientes.

\section{Referencias}

1. United Nations. World contraceptive use 2007 (wall chart). New York: United Nations, Department of Economic and Social Affairs, Population Division; 2008.

2. Population Reference Bureau. Family planning worldwide 2008 data sheet. Washington, D.C: Population Reference Bureau; 2008.

3. Schwingl PJ, Guess HA. Safety and effectiveness of vasectomy. Fertil Steril. 2000;73:923-36.

4. Adams CE, Wald M. Risks and Complications of Vasectomy. Urol Clin N Am. 2009;36:331-6.

5. Deneux-Tharaux C, Kahn E, Nazerali H. Pregnancy rates after vasectomy: a survey of US urologists. Contraception 2004;69:401-6.

6. Mansour D, Inki P, Gemzell-Danielsson K. Efficacy of contraceptive methods: A review of the literature. Eur J Contracept Reprod Health Care 2010;5:4-16.

7. Armand Z, Vasectomy update 2010. Can Urol Assoc J. 2010;4:306-9.

8. Hama Attar K, Gurung P, Holden S, Peters J, Philip T. Clearance after vasectomy: Has the time come to modify the current practice?. Scand J Urol Nephrol. 2010;44:147-50.

9. Korthorst R, Consten D, van Roijen JH. Clearance after vasectomy with a single semen sample containing < than 100.000 immotile sperm $/ \mathrm{mL}$ : analysis of 1073 patients. BJU Int. 2009;105:1572-5.

10. Labrecque M, St-Hilaire K, Turcot L. Delayed vasectomy success in men with a first postvasectomy semen analysis showing motile sperm. Fertil Steril. 2005;83:1435-41.

11. De Knijff DW, Vrijhof HJ, Arends J, Janknegt RA. Persistence or reappearance of nonmotile sperm after 
vasectomy: does it have clinical consequences? Fertil Steril. 1997;67:332-5.

12. Dohle GR, Diemer T, Kopa Z, Krausz C, Giwercman A, Jungwirth A. European Association of Urology Guidelines on Vasectomy. Eur Urol. 2012;61:159-63.

13. Li SQ, Goldstein M, Zhu J, Huber D. The no-scalpel vasectomy. J Urol. 1991;145:341-4.

14. Art K, Nangia A. Techniques of Vasectomy. Urol Clin N Am. 2009;36:307-16.

15. Shakeri S, Aminsharifi AR, Khalafi M. Fascial Interposi- tion Technique for Vasectomy: Is It Justified?. Urol Int. 2009;82:361-4.

16. Griffin T, Tooher R, Nowakowski K, Lloyd M, Maddern G. How little is enough? The evidence for post-vasectomy testing. J Urol. 2005;174:29-36.

17. Bhatta Dhar N, Bhatt A, Jones JS. Determining the success of vasectomy. BJU Int. 2006;97:773-6.

18. Trussell J. Contraceptive efficacy. In: Hatcher R, editor. Contraceptive Technology, 18th ed. New York, NY: Ardent Media; 2004. p. 773-845. 